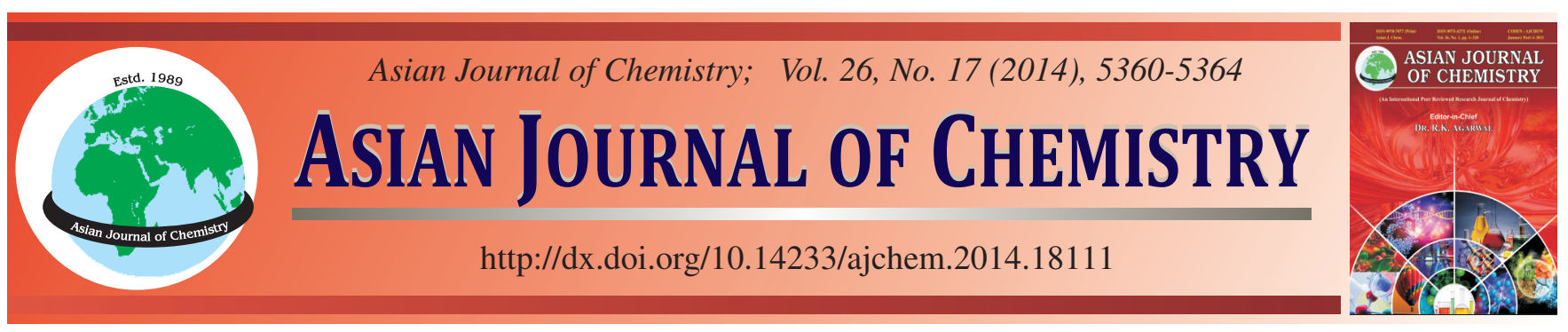

\title{
Atomization Characteristics of Gas-Liquid Two Phase Rotation Flow Spray Nozzle $\dagger$
}

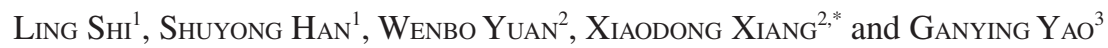

${ }^{1}$ Hubei Key Laboratory of Industrial Fume \& Dust Pollution Control, Janghan University, Wuhan 430056, P.R. China

${ }^{2}$ Hubei Key Laboratory for Efficient Utilization and Agglomeration of Metallurgic Mineral Resources, Wuhan University of Science and Technology, Wuhan 430081, P.R. China

${ }^{3}$ Wuhan Iron and Steel Co. Ltd., Wuhan 430083, P.R. China

*Corresponding author: Tel./Fax: +86 27 68862875; E-mail: ling_s_h@163.com

\begin{abstract}
In order to improve the characteristics of the spray nozzle, a new kind of gas-liquid two-phase rotation flow nozzle is developed. Spray characters and inner velocity distribution for the two-phase rotation flow nozzle are investigated by numerical simulation and experimental measuring, respectively. The simulation results obtained by the computing fluid software, CFX, illustrated that the liquid is changed into a rotary band due to the high gas shear strength. Therefore, the fine water droplets can be easily produced when this two-phase fluid flows to the end of the nozzle. The atomized droplet size is measured by winner 313 spray laser particle size analyzer in the laboratory condition. The experimental data show that the relation of the spray median diameter and the gas-liquid ratio follows a power function. It is helpful to determine the operation parameters for its application in industry.
\end{abstract}

Keywords: Gas-liquid two phase nozzle, Rotation flow, Spray nozzle, Numerical simulation, Gas-liquid ratio.

\section{INTRODUCTION}

Atomization nozzle is widely used in almost all the industrial fields including the sources of energy, environmental protection, chemical industry, agriculture, construction, mining, metallurgical industry, power industry etc. ${ }^{1}$. Atomization nozzle has especially played an important role in the air pollution system, such as in the wet dust control system and the wet desulfurization system.

There are two kinds of nozzle mainly: one is the air-stream atomization nozzle and the other is "solid" or "airless" injection atomization $^{2}$. The quality of a nozzle is characterized by the diameter distribution of droplets, flow rate, operating pressure, spray pattern, quality of atomization, material of manufacture etc. ${ }^{3,4}$.

The gas-liquid two-phase atomization nozzle is an airstream nozzle. Liquid is atomized in this nozzle due to the air at high speed passing over the surface of a liquid. It is found that, when the relative air speed is sufficiently high, the liquid is broken into minute droplet ${ }^{2,3,5}$. It has several advantages, such as better atomization effect, smaller gas-liquid-ratio and simpler structure. However, the instability of the atomization quality and the block of nozzle orifice are disadvantageous to its use in the pollution control, especially in the case of the flue gas desulfurization due to the high dense of limestone slurry $^{6}$. Therefore, the desulfurization spray nozzle has received considerable attention over the years ${ }^{1,7}$. Wang et al. ${ }^{8}$ examined the atomization characteristics of an electro-hydro-dynamics limestone water spray and observed the changes in the droplet diameter and the specific charge in relation to various applied voltages. Cheng et al. ${ }^{9}$ examined the relationship between nozzle spray pressure and atomization particle size.

In this work, firstly, the internal fluid field of a new kind of gas-liquid two-phase rotation flow nozzle (referred to as the rotation flow nozzle $)^{10}$, which is developed for the ammonia simultaneous desulfurization and de-nitrification process of the iron, steel sintering flue gas and dust control, is analyzed by numerical simulation to reveal the internal velocity of gas and liquid. Then, the experiments are conducted as to the diameter of the droplet versus the gas-liquid-ratio. The objective is to find a guide for its engineering application.

Structure and principle of the rotation flow nozzle: The structure of the rotation flow nozzle is schematically sketched in Fig. 1. It consists of a center liquid inlet pipe, a side gas inlet pipe, a vortex tube, a circular seam and orifices. The nozzle is made from stainless steel and the parameters of the nozzle are given in Table- 1 . 


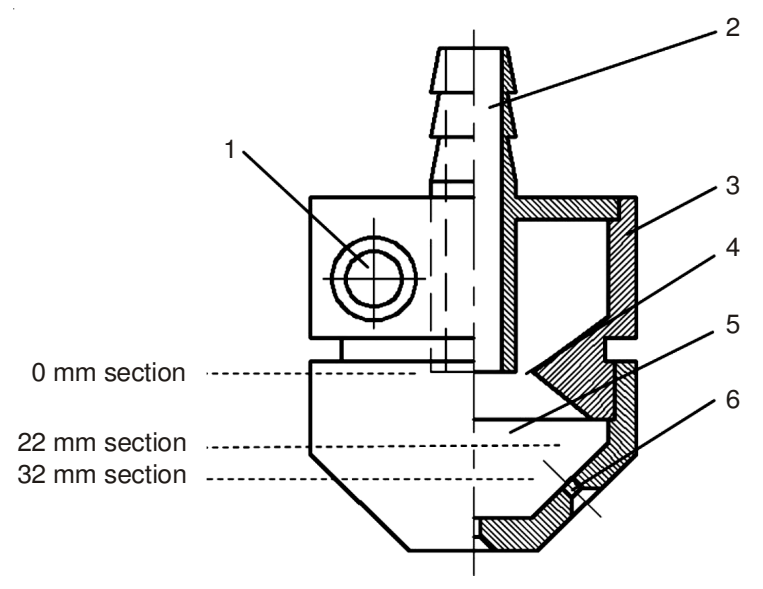

Fig. 1. Schematic diagram of the nozzle structure

\begin{tabular}{lcc}
\multicolumn{3}{c}{ TABLE-1 } \\
\multicolumn{3}{c}{ PARAMETERS OF THE NOZZLE } \\
\hline \multicolumn{1}{c}{ Item } & Unit & Value \\
\hline Jet angular & $\mathrm{o}$ & $100-120$ \\
Orifice diameter series & $\mathrm{mm}$ & $2,3,4$ \\
Number of orifice & $\mathrm{piece}$ & 9 \\
Maximum gas pressure & $\mathrm{MPa}$ & 0.3 \\
Maximum water pressure & $\mathrm{MPa}$ & 0.2 \\
Maximum rate of flow of water & $\mathrm{L} / \mathrm{h}$ & 500 \\
\hline
\end{tabular}

The film splitting is a main mechanism of the rotation flow nozzle. The process of spray for the rotation flow nozzle is: the compressed air jet in the nozzle through the tangential inlet pipe, the gas accelerates in the vortex tube due to the unique structure of the inlet pipe. The maximum velocity of gas is at $0 \mathrm{~mm}$ cross-section where is a circular seam. The velocity of gas may reach a speed of super-sonic. So there is a bigger negative pressure, which let the water enter in the circular seam at high velocity, at the exit of liquid. When the gas and liquid face with at high relative speed at circular seam, the liquid flow is strongly sheared and rushed into very small water droplets which wrapped the air bubble and the gas-liquid mixture flow occur and mix in the mixing chamber. The first atomization finishes. Next, the gas-liquid mixture flow gets decelerating and expanding, the air bubble 'blast' makes the droplet atomization in the mixing chamber. The second atomization occurs. Finally, the mixture flow ejects from the end of Venturi orifice meeting with the air. The third atomization occurs. The outstanding advantage of the nozzle is that the functions of the high-speed spiral airflow, the liquid film and the ability of droplet fracture are combined altogether. The rate of liquid may continuously change with the adjustment of gas pressure.

Numerical simulation for the rotation flow nozzle: It is known that the large velocity difference between gas and liquid is a key factor. Since it is difficult to monitor the velocity of gas or liquid in a nozzle, the commercial software CFX is used to simulate the internal streamline distribution, the velocity distribution of the gas-liquid two-phase nozzle.
Structured grid of the nozzle: A 9-orifice nozzle is taken as example for the numerical simulation of the fluid field here. The geometric model of the nozzle is established according to its actual size (the wall thickness ignored) with the help of Auto CAD 3D drawing software. The pretreatment is conducted using the ICEM for meshing. The whole computational domain is divided into 691877 elements to meet the needs of the numerical calculation precision and the calculation efficiency. Fig. 2 shows the grid of the nozzle. Table- 2 gives the quality of mesh.

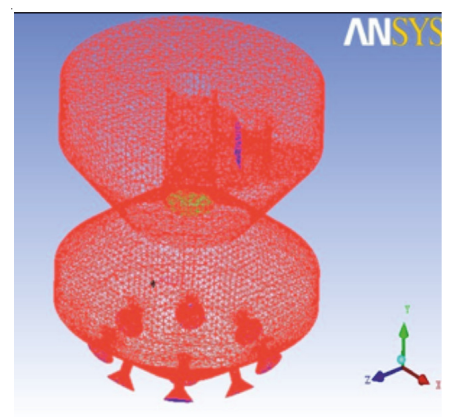

Fig. 2. Unstructured grid of the nozzle

Numerical model and boundary conditions: Here, the RNG k- $\varepsilon$ model is adopted for numerical simulation directly, the control equations as follows ${ }^{11-14}$ :

$$
\begin{gathered}
\frac{\partial(\rho \mathrm{k})}{\partial \mathrm{t}}+\frac{\partial\left(\rho \mathrm{ku}_{\mathrm{i}}\right)}{\partial \mathrm{x}_{\mathrm{i}}}=\frac{\partial}{\partial \mathrm{x}_{\mathrm{j}}}\left[\alpha_{\mathrm{k}} \mu_{\mathrm{eff}} \frac{\partial \mathrm{k}}{\partial \mathrm{x}_{\mathrm{j}}}\right]+\mathrm{G}_{\mathrm{k}}+\rho \varepsilon \\
\frac{\partial(\rho \varepsilon)}{\partial \mathrm{t}}+\frac{\partial\left(\rho \varepsilon \mathrm{u}_{\mathrm{i}}\right)}{\partial \mathrm{x}_{\mathrm{i}}}=\frac{\partial}{\partial \mathrm{x}_{\mathrm{j}}}\left[\alpha_{\varepsilon} \mu_{\mathrm{eff}} \frac{\partial \varepsilon}{\partial \mathrm{x}_{\mathrm{j}}}\right]+\frac{\mathrm{C}_{1 \varepsilon}^{*}}{\mathrm{k}} \mathrm{G}_{\mathrm{k}}-\mathrm{C}_{2 \varepsilon} \rho \frac{\varepsilon^{2}}{\mathrm{k}} \\
\text { where, } \mu_{\mathrm{eff}}=\mu+\mu_{\mathrm{t}}, \mu_{\mathrm{t}}=\rho \mathrm{C}_{\mu} \frac{\mathrm{k}^{2}}{\varepsilon} \\
\mathrm{C}_{\mu}=0.0845, \alpha_{\mathrm{k}}=\alpha_{\varepsilon}=1.39, \mathrm{C}_{1 \varepsilon}^{*}=1.42, \mathrm{C}_{2 \varepsilon}=1.68 \\
\eta=\sqrt{2 \mathrm{E}_{\mathrm{ij}} \cdot \mathrm{E}_{\mathrm{ij}}} \frac{\mathrm{k}}{\varepsilon}, \mathrm{E}_{\mathrm{ij}}=\frac{1}{2}\left(\frac{\partial \mathrm{u}_{\mathrm{i}}}{\partial \mathrm{x}_{\mathrm{j}}}+\frac{\partial \mathrm{u}_{\mathrm{j}}}{\partial \mathrm{x}_{\mathrm{i}}}\right)
\end{gathered}
$$

$\eta_{0}=4.377, \beta=0.012$

The entrance boundaries of the nozzle are set, respectively at the liquid pipe inlet and air pipe inlet, which are kwon as velocity boundary condition. The flow rate of liquid is $500 \mathrm{~L} / \mathrm{h}$ and the ratio of the gas-to-liquid is $0.006 \mathrm{~kg} / \mathrm{kg}$. The outlet boundary, which is kwon as pressure boundary condition, is set as an atmospheric pressure with the environmental temperature of $25^{\circ} \mathrm{C}$.

Numerical simulation result: Fig. $3 a-b$ are internal gas and liquid streamline diagram of the nozzle, respectively. The airflow is running at the speed of 10 times of the liquid. Fig.3a shows that the gas gets into the nozzle through the inlet pipe

\begin{tabular}{ccccccccccc}
\multicolumn{10}{c}{ TABLE-2 } \\
\multicolumn{10}{c}{ QUALITY OF MESH } \\
\hline Quality indicators & $0.0-0.1$ & $0.1-0.2$ & $0.2-0.3$ & $0.3-0.4$ & $0.4-0.5$ & $0.5-0.6$ & $0.6-0.7$ & $0.7-0.8$ & $0.8-0.9$ & $0.9-1.0$ \\
\hline Percent & $0 \%$ & $0 \%$ & $0.019 \%$ & $0.627 \%$ & $3.799 \%$ & $5.769 \%$ & $6.77 \%$ & $8.013 \%$ & $9.731 \%$ & $65.273 \%$ \\
\hline
\end{tabular}




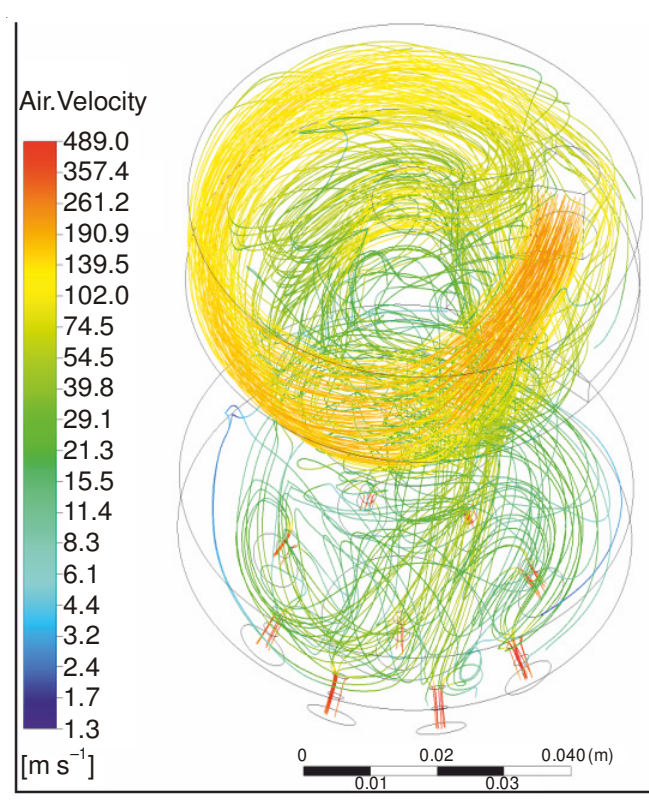

(a)

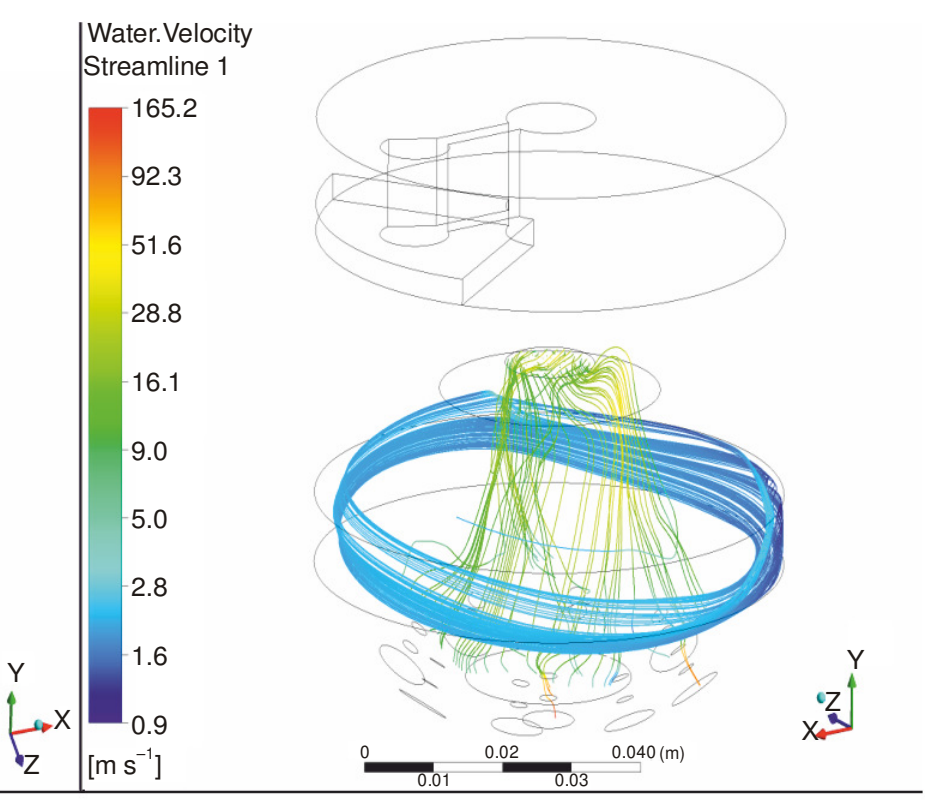

(b)

Fig. 3. Internal gas and liquid streamline diagram of the nozzle (a) gas streamline; (b) liquid streamline

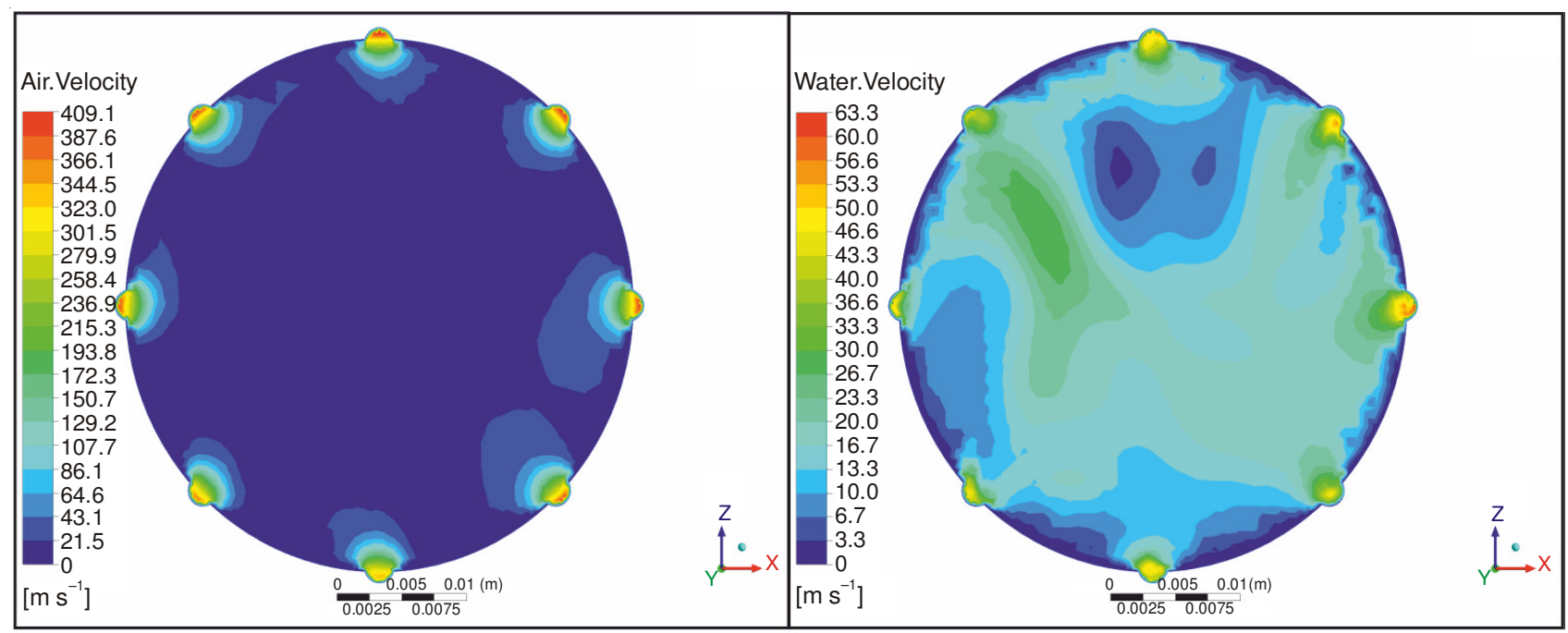

(a)

(b)

Fig. 4. Isokinetic velocity cloud of gas and liquid at $32 \mathrm{~mm}$ section (a) gas streamline; (b) liquid streamline

to forms rapidly downward rotating airflow. As Fig 3b shows, the liquid flow in the mixing chamber gets twisted, rushed and dispersed under the influence of the high gas shear strength. So, as the result of that, the liquid flow changes into a rotary band and become low-speed annular flow in the mixing chamber. This gas-liquid two phase distribution is very beneficial to the uniform dispersion of the droplets.

Fig. 4a-b show isokinetic cloud of gas and liquid at the interface (the $32 \mathrm{~mm}$ cross-section in Fig. 1) of the mixing chamber and the orifice, respectively. As shown Fig. 4a, the volume of the gas begins to expand rapidly around the orifices. There is a high velocity difference between the gas and the liquid. The maximum speed of gas is $409.1 \mathrm{~m} / \mathrm{s}$ in Fig 4a, while the maximum speed of the liquid is $63.3 \mathrm{~m} / \mathrm{s}$ in Fig. $4 \mathrm{~b}$ by numerical analysis. It is obvious that the higher velocity difference is, the better atomization effect can be obtained.

\section{RESULTS AND DISCUSSION}

The experimental system mainly consists of an air compressor, a gas tank, a water supply tank, two flow meters, a rotation flow nozzle, a laser measuring device and a computer, as shown in Fig. 5.

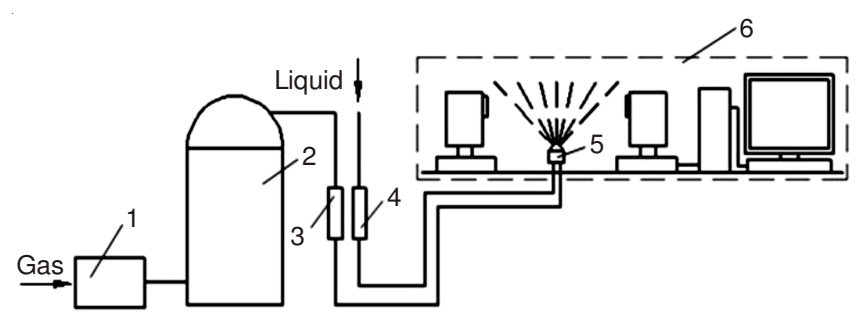

Fig. 5. Schematic diagram of the experimental system. 1-Air compressor 2-gas tank 3-gas flow meters 4-liquid flow meters 5-rotation flow nozzle 6-spray laser measuring system 
Water and compressed air are used as the medium in the experiment. Winner 313 laser particle size analyzer is used to measure the spray median diameter distribution. At first, supply water and compressed air to the nozzle, respectively. And then, regulate the flow of water and gas to the settled gas-liquid ratio. Finally, the spray median diameter distribution will automatically be measured by the laser particle size analyzer when the fine water droplets spray through the central between the transmitter and the receiver.

Fig. 6 shows the spray median diameter distribution at the gas-liquid ratio of $50 \mathrm{~m}^{3} / \mathrm{m}^{3}$ when the nozzle is $20 \mathrm{~cm}$ from the center of the laser analyzer at $4 \mathrm{~mm}$ orifice diameter at 0.1 Mpa gas pressure. It can be read directly from the figure that the spray median diameter is $96 \mu \mathrm{m}$. Similarly, as to regulate the air flow at different rates of $5,7.5,10,12.5$ and $15 \mathrm{~m}^{3} / \mathrm{h}$, respectively, the different spray median diameter distributions can be measured.

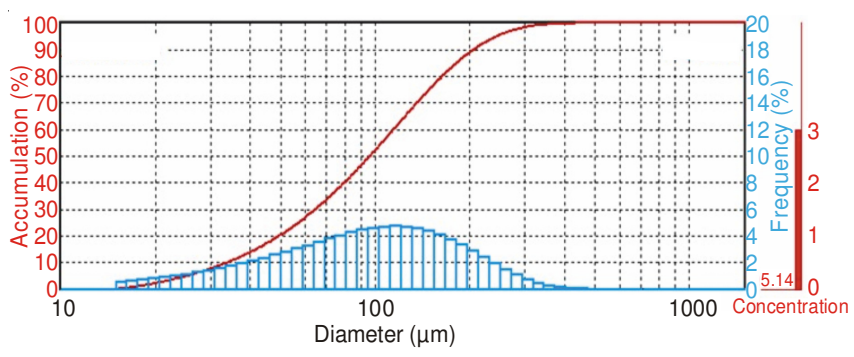

Fig. 6. Atomized water droplet size distribution at the gas-liquid ratio of $50 \mathrm{~m}^{3} / \mathrm{m}^{3}$

Table- 3 gives the experimental results of the spray median diameter under different gas-liquid ratio. It is easy to figure out that the spray median diameter decreases with the increasing of the gas-liquid ratio. In order to determine the operation parameters in industrial application, the characteristic mathe-matical model of the nozzle is needed to be set up according to the experimental data. The fitting equation is expressed as

\section{TABLE-3}

EXPERIMENTAL RESULTS OF THE SPRAY MEDIAN DIAMETER UNDER DIFFERENT GAS-LIQUID RATIO

\begin{tabular}{cc|cc}
\hline $\begin{array}{c}\text { Gas-liquid } \\
\text { ratio, } \mathrm{x}\left(\mathrm{m}^{3} / \mathrm{m}^{3}\right)\end{array}$ & $\begin{array}{c}\text { Spray median } \\
\text { diameter, } \mathrm{y} \\
(\mu \mathrm{m})\end{array}$ & $\begin{array}{c}\text { Gas-liquid } \\
\text { ratio, } \mathrm{x}\left(\mathrm{m}^{3} / \mathrm{m}^{3}\right)\end{array}$ & $\begin{array}{c}\text { Spray median } \\
\text { diameter, } \mathrm{y} \\
(\mu \mathrm{m})\end{array}$ \\
\hline 25.0 & 280.79 & 75.0 & 40.02 \\
37.5 & 169.37 & 100.0 & 42.46 \\
50.0 & 95.88 & 125.0 & 45.88 \\
62.5 & 45.35 & - & - \\
\hline
\end{tabular}

$$
y=14471 x^{-1.28}
$$

The spray median diameter may be estimated by eqn. 3 for engineering application in desulfurization. The experimental data and the fitting curve are illustrated in Fig. 7. It is shown that the variation between the droplet size and the gasliquid ratio can be desirably described by power function. Therefore, it is convenient to select the proper gas-liquid ratio according to the fitting curve displayed in Fig. 7 if a particular droplet size is needed in practice.

As a comparison, the spray median diameter for a $3 \mathrm{~mm}$ orifice diameter nozzle is measured under other parameters.

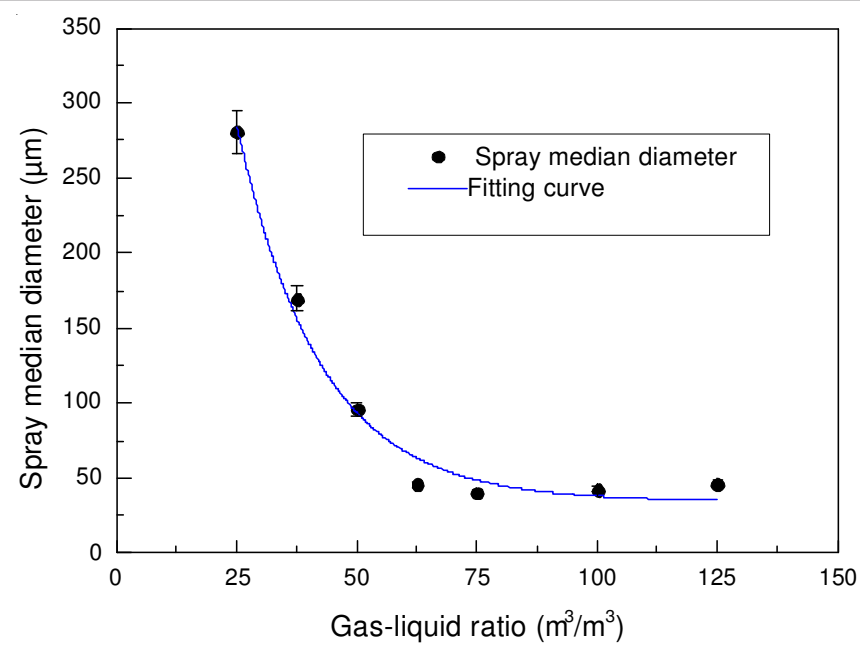

Fig. 7. Relation of spray median diameter and gas-liquid ratio

The experimental condition is shown in Fig. 8. A comparison with Fig. 7, it is clear that the spray median diameter is smaller at high gas pressure and fine diameter of a orifice.

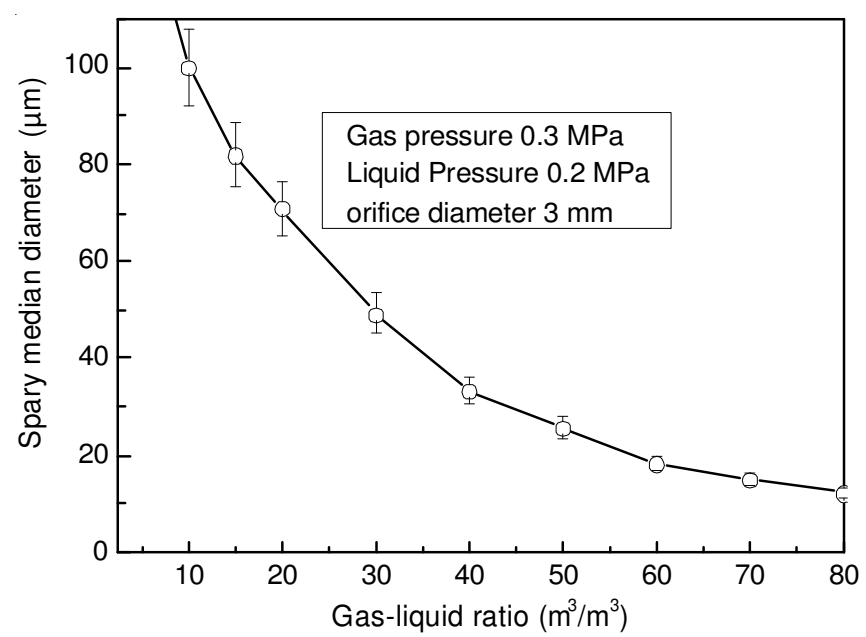

Fig. 8. Spray median diameter at maximum gas pressure and water pressure

\section{Conclusion}

It is found that the liquid flow has been sufficiently atomized in the mixing chamber of the nozzle due to the high gas shear strength according to the simulation analysis of the gas-liquid two phase streamline diagram and the isokinetic velocity. Furthermore, near the orifices, the water droplets can be further atomized due to the rapid decrease cross-sectional area of the orifices and the speed of flow increases. The simulation indicates that the velocity of the droplet near the orifices can reach $489 \mathrm{~m} / \mathrm{s}$ that is higher than the speed of sound.

The big orifice diameter (with a diameter of $2-5 \mathrm{~mm}$ ) design is adopted. The possibility of being blocked can be greatly reduced. Since the liquid flow has been sufficiently atomized in the mixing chamber, it is possible to enlarge the diameter of the orifice further without affecting the atomization seriously.

There is typical power function relation between the median diameter of the water droplets and the gas-liquid ratio. It is known that extremely small droplets are not suitable for smoke purification. In this case, the best droplets median 
diameter is between $100-300 \mu \mathrm{m}^{6}$. Fig. 7 implies that the gasliquid ratio is between $20-60 \mathrm{~m}^{3} / \mathrm{m}^{3}$. This range of the gasliquid ratio is quite wide. It means that this rotation flow nozzle has good atomizing stability for industrial application.

\section{ACKNOWLEDGEMENTS}

The authors thank Hubei Key Laboratory of Industrial Fume \& Dust Pollution Control open project (HBIK2012-01), Wuhan Science and technology project (2013061001010482) and National High Technology Research and Development Program of China (863 Program, No. 2012AA062501 ) for supporting this study.

\section{REFERENCES}

1. H. Lingyun and H. Xiaochun, Technical Manual of Nozzle, Sinopec Press, Beijing (2007).

2. R.D. Reitz and F.V. Bracco, Phys. Fluids, 25, 1730 (1982).
3. L.-J. Yang, W. Wang, Z.-B. Hu, and Z.P. Zhang, J. Beijing Univ. Aeronautics Astronautics, 413 (2002).

4. W.F. Wu, Q.K. Feng, Q.J. Xiang and J.X. Lu, Nuclear Power Eng., 28, 34 (2007).

5. Z. Feras, Batarseh, L. Lia, V. Roisman and C. Tropea, ILASS Americas, $21^{\#}$ Annual Conference on Liquid Atomization and Spray System, Orlando, Florida, pp. 18-21 (2008).

6. P.R. Hu, L. Hui and Y.Q. Wang, J. Filtr. Sep., 21, 20 (2011).

7. Y. Zhou, M. Zhang, D. Wang and L. Wang, Ind. Eng. Chem. Res., 44, 8830 (2005)

8. S.H. Wang, J.S. Chang and A.A. Berezin, J. Electrostatics, 30, 235 (1993).

9. W.-M. Cheng, G. Zhou, Q.-M. Zuo, W. Nie and G. Wang, J. China Coal Soc., 35, 1308 (2010).

10. X.D. Xiang, 2009100631563 (2009).

11. W. Houqing, S. Chao and X.J. Wang, China Petrol. Machinery, 33, 9 (2005).

12. B. Dobrowolski, K. Krècisz and A. Spyra, Task Quarterly, 4, 439 (2005).

13. S.A. Orszag, J. Sci. Comput., 1, 1 (1986).

14. Q. Chen, Numerical Heat Transfer B-Fundamentals, 28, 353 (1995). 